that the filtrates from the above precipitates remained perfectly clear for periods of from thirty to ninety minutes, varying with the temperature at which they were kept, is worthy of note.

It is therefore clear that all experimental evidence thus far points to the correctness of Recoura's peculiar symbols representing chromium compounds, for here in the case of the chrome alum solution, the free acid, as well as the directly ionizable sulphuric acid radicals, has been shown to correspond in quantity with the requirements of the symbol,

$$
2 \mathrm{~K}_{2} \mathrm{SO}_{4} ; \mathrm{Cr}_{4} \mathrm{O}\left(\mathrm{SO}_{4}\right)_{4} \mathrm{SO}_{4} ; \mathrm{H}_{2} \mathrm{SO}_{4}
$$

$i . e$, one-half of the $\mathrm{SO}_{4}$ exists as ordinary sulphate, including one-eighth as free acid, and the other half in a condition of considerable stability and non-precipitable, at ordinary temperatures.

LABORATORY OF THE MASSACHUSETTS INSTITUTE OF TECHNOLOGY,

\title{
THE DETERMINATION OF THE BROMINE ABSORPTION OF OILS.
}

BY PARKER C. MCILHINEY.

Receired September 22, 1890 .

SOME time ago the writer suggested a method of fat analysis, the determination of the unsaturated constituents of fats and oils.' Since then Hehner has called attention to the fact that Allen ${ }^{3}$ had previously described a similar method of analysis for shale oils. This fact had escaped the writer's attention and his apologies are due to $\mathrm{Mr}$. Allen for the omission of credit where it belonged.

Further experience with the method has confirmed the belief that its results are more valuable than those given by the $\mathrm{Hibl}$ process, and recently it has been found possible to so modify its details that it can be carried out more easily and expeditiously. The method consists briefly in adding to a weighed portion of the fat or resin in a stoppered bottle, an excess of standard solution of bromine in carbon tetrachloride, and, after the reaction between them has taken place, determining the excess of bromine

1 This Journal, $26,275$.

2 Analyst, 20, 40 (1895).

3 Commercial Organic Analysis, second edition, 2, $3 S_{3}$. 
by adding an aqueous solution of potassium iodide and titrating with sodium thiosulphate. Any hydrobromic acid which may have been formed in the reaction is determined in the aqueous solution. The percentage of bromine found as hydrobromic acid is called the bromine substitution figure, and the total percentage of bromine absorbed, less twice the bromine substitution figure, gives the bromine addition figure.

When the original article was written the rapidity with which bromine reacts with fats was not appreciated. On investigation it was proved that if the fat is treated with double the quantity of bromine with which it can combine, the reaction between them is complete within one minute; no higher addition figures were obtained when the tests were continued for a number of hours. This agrees with the statement of Hehner and Mitchell that the reaction is instantaneous. The process can therefore be carried out very rapidly.

Several objections to the $\mathrm{Hubl}$ process may be stated as follows:

I. The solution of iodine and mercuric chloride is not a stable one. When freshly prepared, its action is apt to be erratic for the first few hours and after that its strength steadily decreases. The result of this is that several hours must be devoted to the preparation of the reagent before the analysis proper can be carried on.

2. The reaction between the reagent and oil is not instantaneous; in fact several hours are required for its completion. With drying oils probably eighteen hours are required. This further delays the analysis.

3. The process does not distinguish between iodine absorbed by addition and that absorbed by substitution. In the case of pure glycerides this is not so serious a defect as in the case of hydrocarbon oils and resins, which absorb halogen mostly by substitution of the halogen for hydrogen. The time allowed for the reaction affects the amount of halogen absorbed to a much greater extent with the latter class of substances than with glycerides, so that no definite Hübl figures can be given for them except as a time is arbitrarily fixed for the reaction. This defect of the method is very marked in the case of rosin. Another result of the lack of distinction between addition and 
substitution in the Hubl process is that substances whose behavior toward halogen is essentially different, such as rosin and linseed oil, may give the same $\mathrm{Hubl}$ figures.

The superiority of bromine over iodine as a reagent in fat analysis has been shown by many investigations during the last three years. A process devised by Hehner and Mitchell, ${ }^{1}$ in which the heat developed by the reaction between bromine and oils is measured, appears to possess very decided merits on the score of rapidity, especially if a large number of samples are to be examined at one time. This advantage is not apparent, however, if only a few samples are to be examined. It is open to one of the same objections as the Hübl process in failing to distinguish between addition and substitution and further the errors of the thermometer reading are multiplied by five and a half in calculating the $\mathrm{Hibl}$ figure. It would seem to the writer, although he has had no personal experience with it, that its advantages in rapidity are also possessed by the method which forms the subject of this paper.

The method formerly employed for determining the bromine substitution figure, was to determine the acidity to methyl orange of the aqueous liquid separated from the carbon tetrachloride after the titration with thiosulphate. Experiments were made to compare the results obtained by using methyl orange and by lacmoid and by determining the acid iodometrically with potassium iodate and iodide. The results of the tests showed that in the case of many oils there was little or no difference between the figures obtained. In the case of others, however, the endreaction with methyl orange was indistinct, and lacmoid gave high figures. This is undoubtedly due to the presence of fatty acids in the solution. The iodometric determination in the same way as used by Schweitzer and Lungwitz, ${ }^{2}$ is therefore to be recommended, especially as it involves no separation of the aqueous portion from the carbon tetrachloride and the same thiosulphate solution serves for determining both the excess of bromine and hydrobromic acid.

The process as at present used is as follows: A quantity of the oil to be analyzed is weighed into a glass-stoppered bottle,

1 A nalyst, 20, 49, 146 (1895) ; J. Soc. Chem. Ind., I6, 87, 309 (1897); This Journa1, 18, 378 $(1896)$.

2 J. Soc. Chem. Ind, 14, I30 (1895). 
ten cc. of carbon tetrachloride added to dissolve the oil, and twenty cc. of third-normal bromine in carbon tetrachloride added from a pipette. It is not found necessary in filling the pipette with bromine solution to use any special arrangement to prevent the introduction of bromine vapor into the mouth. Only a rubber tube is necessary. Another pipette full of solution should be added to ten cc. of carbon tetrachloride, and this blank titrated with thiosulphate to determine the strength of the bromine solution. The test itself need be allowed to stand only one or two minutes before adding twenty to thirty cc. of ten per cent. solution of potassium iodide, the amount necessary depending upon the excess of bromine present. An excess, of course, does no harm. In order to prevent any loss of bromine or hydrobromic acid which would probably occur on removing the stopper of the bottle, a short piece of wide rubber tubing, of the sort used for Gooch crucibles, is slipped over the lip of the bottle so as to form a well around the stopper. It is advisable, also, to cool the bottle by setting it into cracked ice in order to produce a partial vacuum in the interior. Into the well formed by the rubber tubing is poured the solution of potassium iodide and the stopper opened slightly. If the bottle has been cooled with ice the iodide solution will be sucked into the bottle, and if it was not cooled some of the air from the interior of the bottle will bubble through the iodide solution, being thereby washed, and allow the iodide solution to enter the bottle. When sufficient iodide solution has been introduced the bottle is agitated to insure the absorption of the bromine and hydrobromic acid by the aqueous solution. The iodine now present is titrated with tenth-normal sodium thiosulphate and when the titration is finished five cc. of a neutral two per cent. solution of potassium iodate is added. This liberates a quantity of iodine equivalent to the hydrobromic acid formed and on titrating this iodine the bromine substitution figure may be calculated. The solution of potassium iodate should be tested for acidity by adding a measured quantity to a solution of potassium iodide and if any iodine is liberated it should be determined with thiosulphate and a suitable correction introduced into the calculation. The potassium iodide, the thiosulphate solution, and the water used should all be tested to see that they are neutral. 
The reaction between bromine and oil appears to be practically instantaneous as far as the bromine taken up by addition is concerned but it seems likely that substitution is distinctly affected by the length of time that the oil and bromine are allowed to remain in contact. The extent to which time affects the substitution figure has not yet been accurately determined, but it is not likely that in ordinary work any serious error is introduced in this way. Experiments to determine accurately the effect of time upon the substitution figure have been undertaken and will be reported later.

For the process in its present form the following advantages may be claimed:

I. The standard solution of bromine is not subject to change on keeping; therefore it may be kept on hand ready for use at short notice; further, it is very easily prepared.

2. The process requires only such time as is necessary for the weighing and the titration, no time being lost in preparing standard solutions or waiting for completion of the reaction between bromine and oil.

3. The absorption of halogen by addition is determined separately from the absorption by substitution, and thus additional information is gained as to the nature of the substance.

4. The solvent used (carbon tetrachloride) is easily recovered, and bromine is cheap, so that the whole process is much cheaper than the Huibl.

The following figures have been obtained upon samples of various oils which are believed to be representative of their respective classes.

The figures given in the last column, obtained by dividing the calculated bromine figures by the actual bromine addition figures, are intended to represent by the amount that they exceed I, $\infty 00$, the extent to which substitution goes on in the determination of the Hübl figure. Thus I.103 represents that over and above the iodine absorbed by addition; ro.3 per cent. of this amount was absorbed by substitution. 
Raw linseed oil, several

years old, 1 ......... I 66.9

Raw linseed oil, several years old, $2 \ldots \ldots \ldots$..... I57.3

Ráw linseed oil, $3 \ldots \ldots$ I 184.2

" " " 4 ".... I78.6

" " $"$ "

" " "

"

" $\quad$ " $\quad 9, \ldots \ldots, \ldots$

Av. omitting Nos. I and 2 183.8

Boiled linseed oil, I.... I8o.4

$$
\begin{aligned}
& \text { " } " \text { " }
\end{aligned}
$$

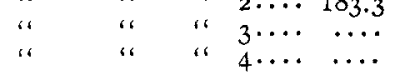

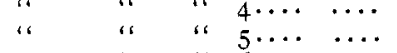

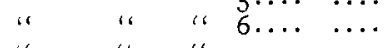

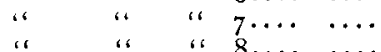

Averages.

$-\cdots$

Petroleum neutrai oil, sp. gr. $0.8436 \ldots \ldots \ldots \ldots$....

Third run rosin oil, I .. $\ldots$

"Mystic" brand rosin oil 63.9

"Java" boiled rosin oil . 73.3

Menhaden oil, I...... $\quad$ I 78.8

$\begin{array}{ccccc}\text { " } & 2 \ldots \ldots \ldots & \text { 1 } 70.4 \\ & & 3 \ldots \cdots \cdots & \text { I } 75.4 \\ & & & \end{array}$

Averages ............ I I 74.9

Corn oil, I $\ldots \ldots \ldots \ldots \ldots, \ldots$

$$
\text { "، } ", 2 \ldots \ldots \ldots \ldots \ldots, \ldots
$$$$
\text { " } \quad \text { " } 3 \ldots \ldots \ldots \ldots, \ldots
$$

Averages ............

Cottouseed oil $\ldots \ldots \ldots \ldots \ldots$

Turpentine ................
Benzine, turps. substitute, I, sp. gr, o.74I ...

Benzine, turps. substitute, 2, sp. gr. $0.733 \ldots \ldots$

Window glass rosin .........

Black rosin $\ldots \ldots \ldots \ldots, \ldots$

$\begin{array}{ll}\text { Ceylon cocoanut oil.... } & \ldots . \\ \text { Tallow ren'd in lab'rat'y } & \ldots .\end{array}$

Tallow ren'din lab'rat'y $\quad \ldots$.
Hard paraffin.......
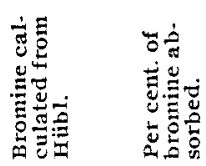

105.2

99. I

II 6 . I

II 2.6

II 7.2

I I $7 \cdot 0$

…

....

-...

I 5.7

II 3.7

I 5.5

$\cdots \cdots$

…

…

$\ldots$

$\ldots$

...

$\cdots$

$\ldots$

.

… I 4.4

$40.3 \quad 92.3$

… 93.7

46.2 IOI.9

II 2.7 I I 4.5

$107.4 \quad 107.3$

IIO. 6

I IO. I

$\overline{\text { II0.2 }} \overline{\text { I } 10.6}$

… 76.2

... 75.8

… 75.4

… $\quad \overline{75.8}$

... 65.8

… 266. I

... 51.5

... 6.3

.... I6I.4

.... $\quad \mathrm{I} 35.4$

$\begin{array}{ll}\ldots . & 5.36\end{array}$

... 24.0

$\ldots$
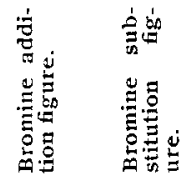

95.4

I. 5

3.6

92.0

IO9. 6

IO2. I

109.2

I06.5

99.9

I09. 5

I09. 4

I06.6

I00.8

105.8

IOI. 2

I03.2

105.2

103.0

I03. 8

IOI.O

103.0

6.4

I6.4

$7 \cdot 7$

6.3
8.3

96.5

92.3

97.9

95.6

73.8

73.2

71.6

72.9

62.2

I66. I

I 5.5

2.7

5.4

4.7

$2 I .48$

3.55

2.8

2.6

2.7

2. 1

3.4

2.3

5.3

2.7

4.9

3.2

90.6

42.3

43.7

46.8

9.0

7.5

7.5

I. 3

I.9

I.5

I. 8

50.0

I8. 0

I. 43

I. 8

76.7

65.0

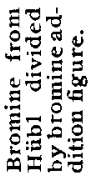

I. $\mathrm{ro}_{3}$

I.000

$3.4 \quad I .059$

3.2 I. 102

$2.0 \quad$ I.072

2.9 I. 098

$2.3 \quad \ldots$

$\ldots$

....

I. 083

2.6 I. 126

2.5 I.09I

....

$\ldots$

$\ldots$

$\ldots$

$\ldots$

$\ldots$

$\ldots$

4.0

$5.23 \mathrm{I}$

5.685

I. 169

I. 164

I. 129

I. I 54

$$
\text { I. } 2
$$

$\cdots$

$\ldots$

$\ldots$

0.33

I. 26

I. 06 